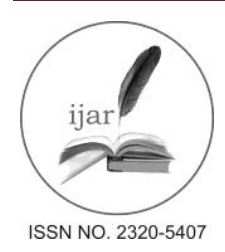

Journal homepage:http://www.journalijar.com

Journal DOI:10.21474/IJAR01

INTERNATIONAL JOURNAL

OF ADVANCED RESEARCH

RESEARCH ARTICLE

\title{
STUDY AND PERFORMANCE OF EZW AND SPIHT ALGORITHM IN SATELLITE IMAGE COMPRESSION.
}

\author{
*Boussaha Abdelkader ${ }^{1}$, and Djebbouri Mohamed ${ }^{2}$. \\ 1. Department of Electronic, Djilali Liabes University, Sidi Bel Abbes, Algeria. \\ 2. Department of Electronic, Djilali Liabes University, Sidi Bel Abbes, Algeria.
}

\section{Manuscript Info Abstract}

Manuscript History:

Received: 15 March 2016

Final Accepted: 22 April 2016

Published Online: May 2016

Key words:

Hyperspectral images, Compression

algorithm,

DCT, EZW, SPIHT.

*Corresponding Author

Boussaha Abdelkader.
In Still images acquired on board the observation satellites of the Earth is more and more characterized by a high spectral resolution with the acquisition of information in the whole spectrum, where traditional remote sensing systems provide some portions of the spectrum. This mode of acquisition brings an assertive quality of data, but do not remain without consequences on systems and conventional equipment transmission and storage, with a cost translates into extra transmission delay and an overflow backup spaces. The primary objective of this work is to answer this double deficit by developing the new satellite image compression techniques. Namely the development of algorithms based on wavelet decomposition image and optimizing said treatment for a better performance in the compression ratio while maintaining image quality developed using both algorithms EZW and SPIHT compared with DCT algorithm.

Copy Right, IJAR, 2016,. All rights reserved.

\section{Introduction:-}

The Earth observation from satellites has been accompanied by a significant increase in the use of satellite or Hyperspectral images in a variety of applications civilian and military (weather, mapping, information, scientific applications, spying ...). The first satellites boarded at their edges the first analogue photography devices posing the problem of retrieving images. A problem quickly erased by the arrival of CCD (Charge Coupled Device),"the advent of digital image was created".Scanning images obtained by the CCD device, is operated by a simple digital to analog conversion ADC to the sensor output. A synchronization of the scanning of the image with the CAN, can sample the picture function in a number of finished items and storing the values thus obtained and then transmit the radio waves. The satellite imagesposed, by their large size, many problems regarding their transmission or their storage. To win both speed in place, the image compression treatment, for the day, has become an indispensable step for increased acquisition capacity. Multiple image compression algorithms have been developed over the years with their complexities and speed flexibility of execution ceased to grow, to achieve a single objective: reduce the size of memory occupied by a picture by losing a minimum of quality.

Among the latest compression techniques implemented in this work, we cite the wavelet transform satellite images using the EZW and SPIHT algorithms aimed to bring a significant progress in improving the compression ratio and PSNR performance compared with image quality maintained. The calculation of lossy DCT domain is much less that of DWT using EZW in termes of comparison. The wavelet-based progressive transmission of image coding and the SPIHT algorithm achieves a high degree of embedding and compression efficiency.

\section{Compression Methods:-}

Our compression scheme is part of an approach to wavelet transform, which is a tool widely used in image processing. Its ability to compact the energy on a small number of coefficients enables efficient sponds to the first 
step of the conventional compression chain and it is commonly used in the case of 2D images by application of separable filters (Mallat algorithm).

For image compression, the transform is the first link in the chain, to compress the information we are going to complete the cycle by quantization and entropy coding. The idea of our scheme of compression adapted to hyperspectral images is represented in Figure 1.

Original

Image

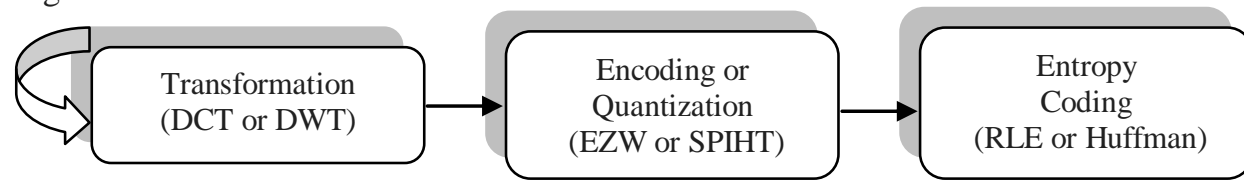

(a)
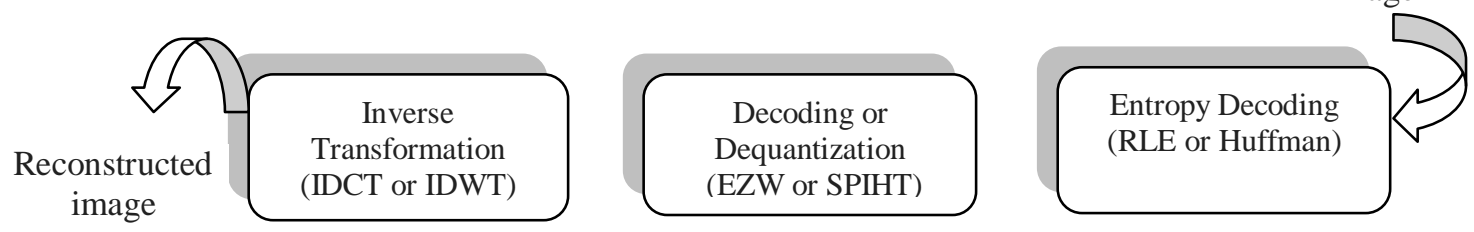

(b)

Fig1 :Diagram of the principle of (a) compression and (b) decompression

\section{DCT Algorithm:-}

The discrete cosine transform (DCT) helps separate the image into parts (or spectral sub-bands) of differing importance (with respect to the image's visual quality). The DCT is similar to the discrete Fourier transform: it transforms a signal or image from the spatial domain to the frequency domain (see Figure 2).

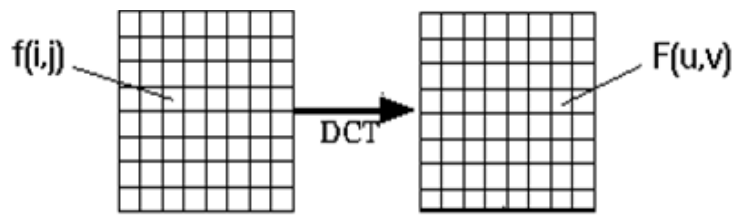

Fig2: Transformation of DCT

Given the difficulty of applying the DCT [1] over the entire matrix, it is decomposed into blocks of size $8 * 8$ pixels. The direct relationship passage of a signal 2D ( $\mathrm{M}$ by $\mathrm{N}$ image) using DCT is defined by the following equation :

$$
F(u, v)=\frac{4 C(u) C(v)}{M N} \sum_{i=0}^{M-1} \sum_{j=0}^{N-1} f(i, j) \times \cos \left[\frac{(2 i+1) u \pi}{2 M}\right] \cos \left[\frac{(2 j+1) v \pi}{2 N}\right]
$$

\section{The basics operations of the DCT algorithm is as follows:}

1. The input spacial image is $\mathrm{N}$ by $\mathrm{M} ; f(i, j)$ is the intensity of the pixel in row $\mathrm{i}$ and column $\mathrm{j}$, and $\mathrm{F}(\mathrm{u}, \mathrm{v})$ is the DCT coefficient in row $u$ and column $v$ of the DCT matrix.

2. The DCT input is an 8 by 8 array of integers. This array contains each pixel's gray scale level;

For most images, much of the signal energy lies at low frequencies; these appear in the upper left corner of the DCT.

3. Compression is achieved since the lower right values represent higher frequencies, and are often small enough to be neglected with little visible distortion. The transformed coefficients are considered representative of the 
spatial frequencies of the original image. DCT provides the best compromise between decorrelation, compaction energy and complexity of implementation.

\section{The Embedded Zero-Tree Wavelet Algorithm (EZW):-}

Shapiro [3] proposed the classic embedded zerotree coding (EZW) using wavelet transform to compress images. Bilgin, Zweig and Marcellin [4]proposed three-dimensional (3D) image compression algorithm with wavelet.

The EZW algorithm implements a progressive, embedded image coding method based on the zerotrees of data structure. All currently significant bits at the same bitplane together and recursively encodes other pixels for the next significant bitplane until reaching the least significant bitplane. As a result, the lower significant bits are embedded behind the higher significant bits, so that a decoder quickly displays a low quality image and better quality as more bits are received.

The EZW encoder exploits the zerotree based on the observation that wavelet coefficients decrease with scale. It assumes that there will be a very high probability that all the coefficients in a quad tree will be smaller than a certain threshold if the root is smaller than this threshold. If this is the case then the whole tree can be coded with a single zerotree symbol. Now if the image is scanned in a predefined order, going from highscale to low, implicitly many positions are coded through the use of zerotree symbols. Of course the zerotree rule will be violated often, but as it turns out in practice, the probability is still very high in general. The price to pay is the addition of the zerotree symbol to our code alphabet (see figure 3).
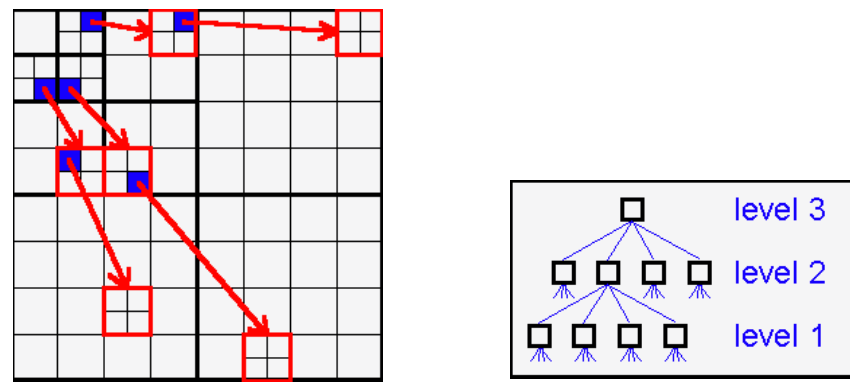

Fig3: The relations between wavelet coefficients in different subbands as quad-trees.

\section{The Steps of the EZW algorithm as follows:}

1. The first step in the EZW coding algorithm is to determine the initial threshold. If we adopt bitplane coding then our initial threshold $\mathrm{T} 0$ will be

$$
\mathrm{T}_{0}=2^{\left(\log _{2}(\operatorname{Max}(|\mathrm{I}(\mathrm{x}, \mathrm{y})|))\right)}
$$

Here $\operatorname{Max}($.$) means the maximum coefficient value in the image and \mathrm{I}(\mathrm{x}, \mathrm{y})$ denotes the coefficients of image.

2. Principal stage. Go through the coefficients on the principal list in an appropriate order and compare each of them with the current threshold Tn. Attribute one of the four following symbols to each coefficient:

$\mathrm{P}$, if it is positive and has an absolute value higher than the threshold;

$\mathrm{N}$, if it is negative and has an absolute value higher than the threshold;

$\mathrm{Z}$, if its absolute value is lower than the threshold but if one of its children has an absolute value higher than the threshold;

$\mathrm{T}$ if its absolute value is lower than the threshold and if all its children have absolute values lower than the threshold.

Thanks to the zerotree property each coefficient that is the child of a coefficient already coded by a $\mathrm{T}$ is not coded because the decoder can determine its value. Each significant coefficient, i.e. of the $\mathrm{P}$ or $\mathrm{N}$ type is placed on the secondary list and is replaced by 0 in the principal list.

The list of symbols is coded in the most efficient manner possible (for example, by a Huffman coding).

3. Secondary stage. For each coefficient I of the secondary list produce 0 or 1 . 
To this end write $d=I(x, y)-\sum_{j \leq n} T_{j}$ and compare $d$ to the middle of the interval $\left[T_{n}, T_{n}+T_{n-1}\right]$. The produced code is 0 if $d \in\left[T_{n}, T_{n}+\frac{1}{2} T_{n-1}\right]$ and 1 if $d \in\left[T_{n}+\frac{1}{2} T_{n-1}, T_{n}+T_{n-1}\right]$

When binary thresholding is applied, testing the position of $d$ is reduced to a comparison of bits. If the paths are numbered starting from 1 and at the pth path of the coefficients it is necessary to extract the (p+1)th bit (starting from the left) from the binary expression of $\mathrm{I}(\mathrm{x}, \mathrm{y})$.

4. New threshold. We read or calculate the new threshold with $T_{n+1}=\left[\frac{1}{2} T_{n}\right]$, for example. If the minimum threshold or the desired compression ratio is attained, we stop; if not, we repeat stages 2, 3 and 4.The Example of application of the EZW algorithm (coding phase) is shown in [7].

\section{The Set PattioningIn Hierarchical Trees Algorithm (SPIHT):-}

The SPIHT algorithm [5] was proposed by Said and Pearlman in 1996 for the 2D image compression with and without loss. This algorithm is based on the same idea as that of Shapiro (EZW) to characterize the dependencies between wavelet coefficients. However, it uses the following three basic principles: a storage part by amplitude of the wavelet coefficients of the 2D DWT, a partitioning in hierarchical trees (each threshold applied trees are sorted on the basis of their meanings in two shaft categories) and scheduling the transmission of refinement bits (the amplitude of each significant coefficient is progressively refined).

\section{The Steps of the SPIHT algorithm as follows:}

1. LIP, LIS and LSP are initialized and the maximum threshold is determined.

2. In the significant pass of the SPIHT algorithm the List of Insignificant Sets (LIS) is examined in regard to the current threshold. With respect to the current threshold the set in the lists are then partitioned into one or more smaller zero tree sets.

3. Isolated insignificant coefficients are appended to the List of Insignificant Pixels (LIP), while significant coefficients are appended to the List of Significant Pixels (LSP).

4. 4) The LIP is also examined and as coefficient become significant with respect to the current threshold, they are appended to the LSP.

5. Binary symbols are encoded to describe motion of sets and coefficients between the three list.

6. Since the list remained implicitly sorted in an importance ordering, SPIHT achieves a high degree of embedding and compression efficiency.

7. For the next scan, threshold is fixed as $T / 2$ and $n$ is $n-1$ and repeat steps 3,4 and 5, until the threshold values or bit rate compliance encoder requirements.

To characterize the parent-child relations in the sub-bands. The following sets of coordinates are used:

$\mathrm{O}(\mathrm{i}, \mathrm{j})$ : All the coordinates of all the children of the node $(\mathrm{i}, \mathrm{j})$.It is expressed in the same way as that of EZW.

$\mathrm{D}(\mathrm{i}, \mathrm{j})$ : All the coordinates of all descendants of the node $(\mathrm{i}, \mathrm{j})$ (A type of zero trees ).

$\mathrm{L}(\mathrm{i}, \mathrm{j})=\mathrm{D}(\mathrm{i}, \mathrm{j})-\mathrm{O}(\mathrm{i}, \mathrm{j})$ : All descendants except for children

(type of tree zeros) (see figure 4).

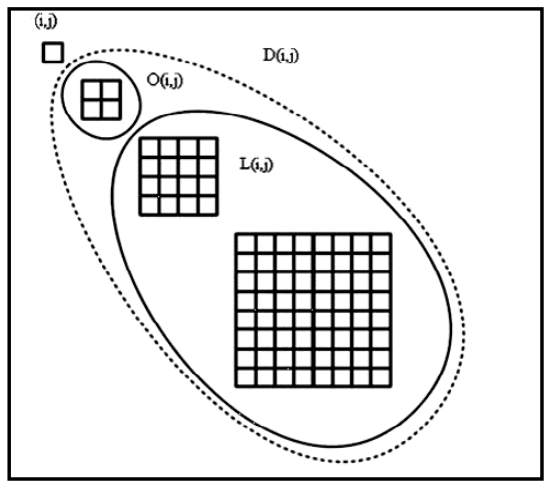

Fig 4:SPIHT Terminology for the descendants. 


\section{Tests and simulation results:-}

Performances measurement :-

\section{A. Compression ratio :-}

The compression ratio (CR) is usually expressed as a percentage. It is measured by the ratio between the volume of the initial image and the compressed image.More compression ratio is high; more the space required for storage decreases ether the time required for transmission.

\section{B. Pick Signal Noise Ratio (PSNR) :-}

$$
C R=\frac{\text { Uncompressed size of original image }}{\text { compressed size of original image }} \times 100 \%
$$

This parameter is expressed by the following relationship:

$$
\operatorname{PSNR}(\mathrm{dB})=10 \log \left[\frac{\mathrm{M} \times \mathrm{N}}{\mathrm{EQM}}\right]
$$

or : $\quad E Q M=\frac{1}{M \times N} \sum_{i=1}^{M} \sum_{j=1}^{N}\left(f_{i, j}-\hat{f}_{i, j}\right)^{2}$

andf $f_{i, j}$ and $\hat{f}_{i, j}$ are the values of the original image and the reconstructed image respectively.

\section{Choice of the test images :-}

For our application, we are used the following test images:

Figure 5 (a) : Lena image $(256 * 256)$ grayscale.

Figure 5 (b) : satellite image of Venis $(1480 * 858) 24$ bit color seen by Pleiades satellite [8].

Figure 5 (c) : satellite image of Toulouse (1772*1772) 24 bit color seen by Pleiades satellite [8].
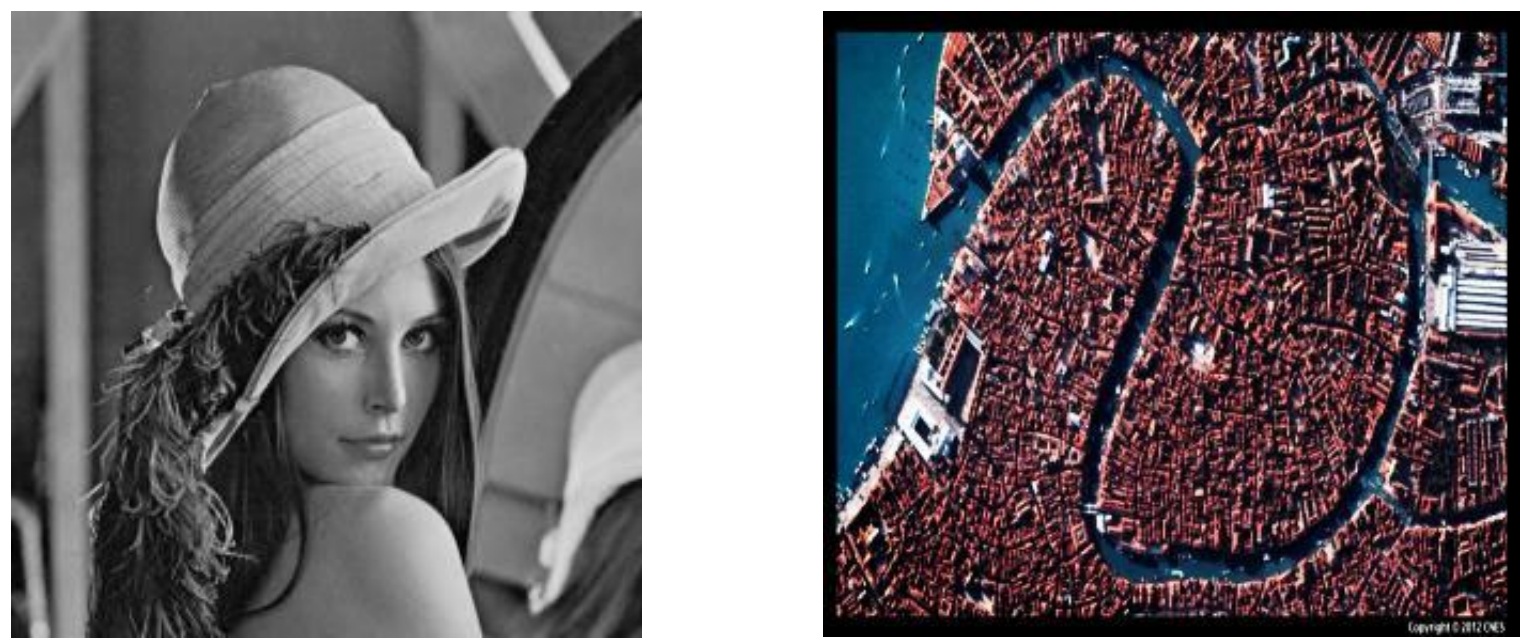

(a)

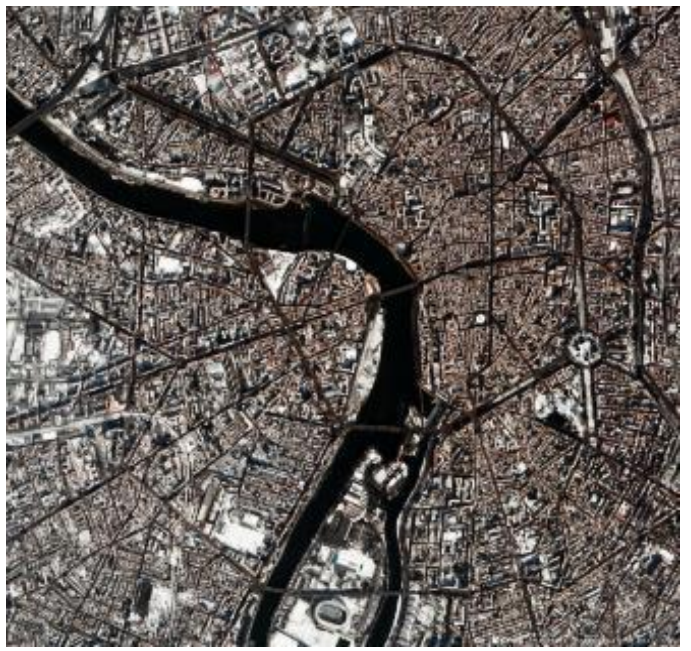

(b)

(c)

Fig 5:TestImages of simulation 


\section{Choice of the Wavelet type :-}

It can be selected in various ways depending on the desired properties of the decomposition base. Among the main properties we note :

1. Regularity : the projection of a mother wavelet function of any lower order polynomial oriented or equal to $\mathrm{P}$ is zero.

2. Perfect reconstruction : the projection of $\mathrm{x}$ on $\mathrm{W}$ family must be reversible.

3. Orthogonality : the basic functions $\mathrm{W}$ are orthogonal.

4. Linear phase : the mother wavelet functions are symmetric or antisymmetric.

In this work, we are use a various types of the wavelet such as, the db8, 10, 12, 16 wavelets, Haar, biorthogonal 2.2 (LeGaLL5/3) and 4.4 (CDF9/7) and Symlet 4, 6, 8,14.

\section{Simulations result and discussion:-}

In this section, we defined the protocol of tests that we used for the validation of our compression method. Relates the metric used : PSNR ,EQM, the compression ratio and the calculation time. We also analyzed the interests of entropy coding (arithmetic) in our coding, finally we finish our study by comparing with some encoders include EZW, SPIHT for wavelet transform with DCT algorithm.

\section{A. Influence of the wavelet type and compression ratio :-}

The following results shows the PSNR valus according to different wavelet type for the compression ratio 50\% (see table 1 and figure 6).

Table 1: PSNR $(\mathrm{dB})$ values for differents wavelets type $(\mathrm{CR}=50 \%)$

\begin{tabular}{cccc}
\hline Wavelet & $\begin{array}{c}\text { Lena } \\
\text { image }\end{array}$ & $\begin{array}{c}\text { Venis } \\
\text { image }\end{array}$ & $\begin{array}{c}\text { Toulouse } \\
\text { image }\end{array}$ \\
\hline Haar & 27.7902 & 36.1254 & 36.0012 \\
HaarN & 27.7880 & 36.1984 & 37.1422 \\
DB4 & 27.7809 & 34.0124 & 37.1227 \\
\hline DB6 & 27.7858 & 35.7789 & 36.1232 \\
\hline DB8 & 28.7797 & 39.0780 & 38.1090 \\
\hline DB14 & 27.7777 & 37.0541 & 37.0277 \\
Sym4 & 27.7759 & 36.8638 & 37.3300 \\
\hline Sym10 & 28.7793 & 38.2014 & 39.4001 \\
\hline Sym14 & 27.7830 & 37.4545 & 36.2255 \\
CDF9/7 & 28.7415 & 40.7100 & 39.9000 \\
\hline LeGaLL5/3 & 28.9731 & 39.4333 & 39.3257 \\
\hline
\end{tabular}

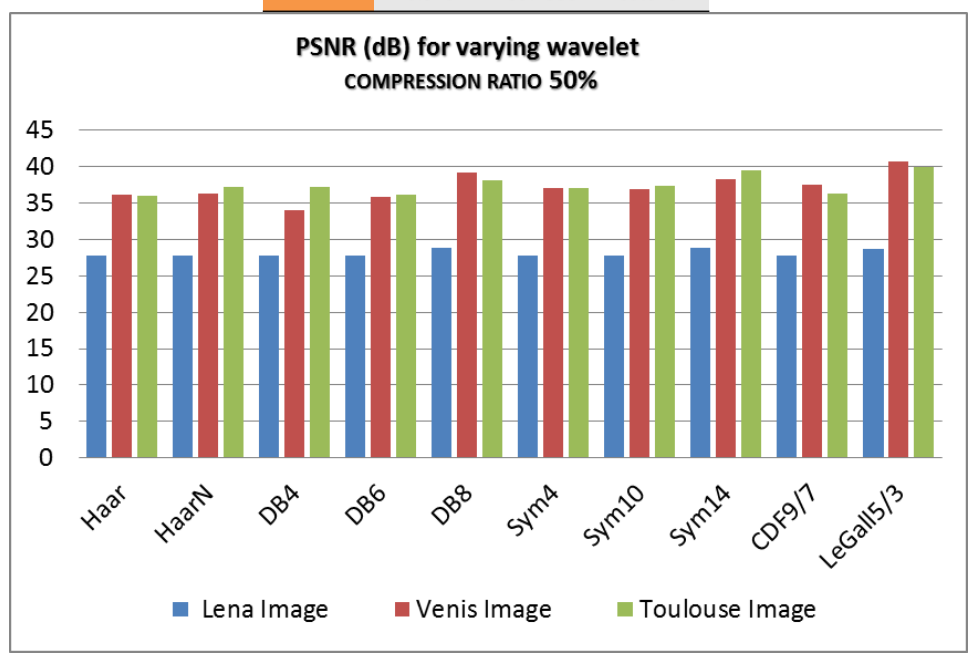

Fig 6: PSNR (dB) values for differents wavelets type $(\mathrm{CR}=50 \%)$ 
The following results shows the PSNR valus according to different wavelet for the compression ratio $70 \%$ (see table 2 and figure 7 ).

Table 2:PSNR (dB) values for differents wavelets type $(\mathrm{CR}=70 \%)$

\begin{tabular}{cccc}
\hline Wavelet & $\begin{array}{c}\text { Lena } \\
\text { image }\end{array}$ & $\begin{array}{c}\text { Venis } \\
\text { image }\end{array}$ & $\begin{array}{c}\text { Toulouse } \\
\text { image }\end{array}$ \\
\hline Haar & $\mathbf{2 7 . 7 2 8 1}$ & 35.2500 & $\mathbf{3 4 . 7 1 5 2}$ \\
HaarN & $\mathbf{2 7 . 7 2 8 6}$ & $\mathbf{3 5 . 0 1 9 3}$ & $\mathbf{3 6 . 7 7 3 1}$ \\
DB4 & $\mathbf{2 7 . 7 2 3 2}$ & $\mathbf{3 4 . 1 2 7 2}$ & $\mathbf{3 6 . 4 4 7 0}$ \\
DB6 & $\mathbf{2 7 . 7 2 3 6}$ & $\mathbf{3 5 . 1 9 8 7}$ & $\mathbf{3 6 . 0 0 4 7}$ \\
DB8 & $\mathbf{2 8 . 7 2 2 7}$ & 39.1644 & 37.2928 \\
DB14 & $\mathbf{2 7 . 7 2 0 8}$ & 35.5531 & 35.0276 \\
Sym4 & $\mathbf{2 7 . 1 2 2 0}$ & $\mathbf{3 6 . 0 0 1 4}$ & $\mathbf{3 6 . 4 0 9 2}$ \\
Sym10 & $\mathbf{2 8 . 7 2 5 0}$ & 38.0045 & 39.2077 \\
Sym14 & $\mathbf{2 7 . 7 2 4 6}$ & 37.1127 & $\mathbf{3 4 . 0 7 0 0}$ \\
CDF9/7 & $\mathbf{2 8 . 5 4 7 2}$ & $\mathbf{4 0 . 5 1 4 0}$ & 39.032 \\
LeGaLL5/3 & $\mathbf{2 8 . 7 7 3 1}$ & 39.0047 & 38.6872 \\
\hline
\end{tabular}

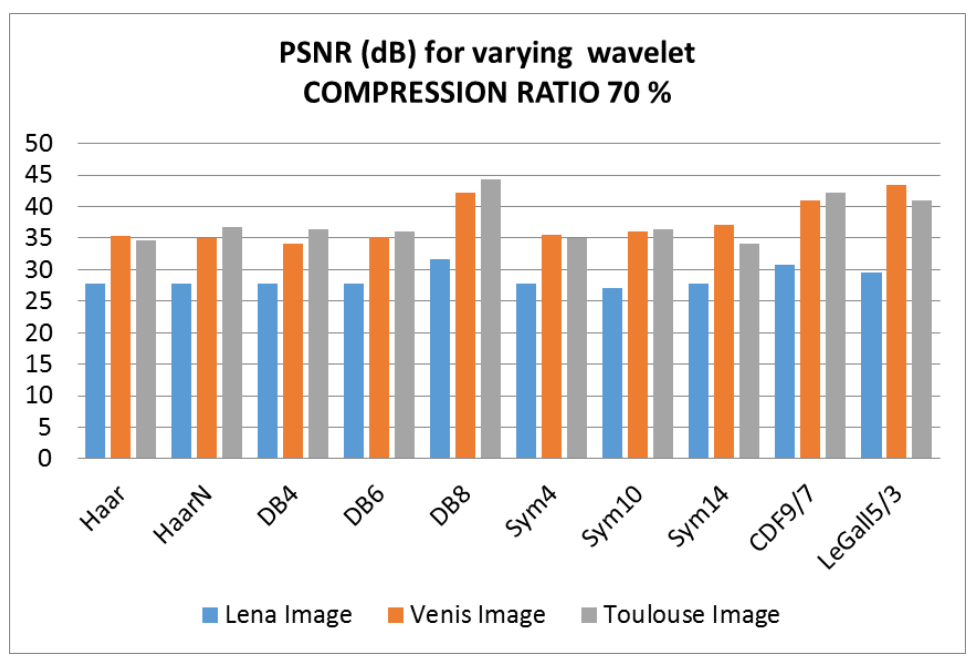

This simulation results show that the PSNR obtained for the satellite imagery (Venis and Toulouse) exceed $35 \mathrm{~dB}$ even at high compression ratios $(\mathrm{CR}=70 \%)$ and the best quality is for the CDF9/7 wavelet type.

\section{B. Influence of the decomposition level :-}

We setting us the choice of the image on the Venis satellite image, then we select the test setup for this new analysis, indeed the choice of dB8 and Sym10 wavelet and EZW as to the quantification method, ether the compression method is setting with Huffman algorithm. The following results shows the valus of PSNR (dB) for the two types of wavelet (dB8 and Sym10) according to differents valus of the decomposition level (see table 3 and figure 8).

Table 3:PSNR (dB) according to the decomposition deph $(\mathrm{CR}=50 \%)$

\begin{tabular}{lll}
\hline & DB8 & Sym10 \\
\hline $\mathrm{J}=1$ & $\mathbf{3 9 . 0 7 8 0}$ & $\mathbf{3 8 . 2 0 1 4}$ \\
$\mathrm{J}=2$ & $\mathbf{3 8 . 8 9 0 2}$ & $\mathbf{3 4 . 1 4 9 8}$ \\
$\mathrm{J}=3$ & $\mathbf{4 3 . 4 3 1 0}$ & $\mathbf{4 5 . 1 8 0 9}$ \\
$\mathrm{J}=4$ & $\mathbf{2 7 . 9 1 7 4}$ & $\mathbf{2 8 . 9 5 8 3}$ \\
$\mathrm{J}=5$ & $\mathbf{2 2 . 5 2 2 8}$ & $\mathbf{2 5 . 3 2 5 1}$ \\
\hline
\end{tabular}




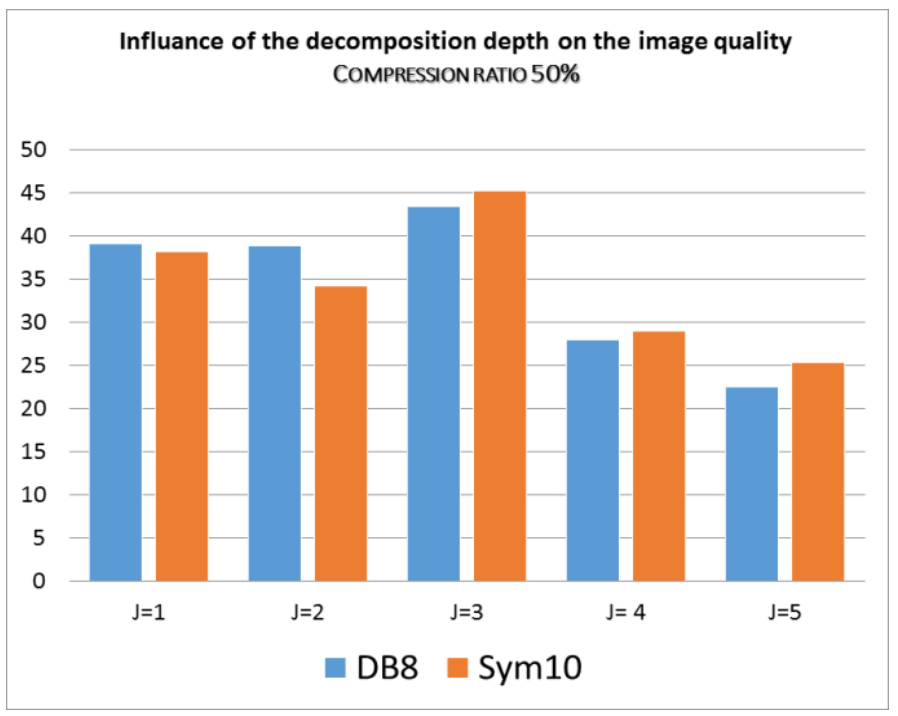

Fig 8: PSNR $(\mathrm{dB})$ according to the decomposition deph $(\mathrm{CR}=50 \%)$

The results obtained show that increasing the number of decomposition levels is obtained a marked improvement in results and this is due to the concept of zero trees which are based on the EZW methods 2D versions. In fact, by increasing the number of decompositions, this enables a better concentration of the useful information in a small number of coefficients in the low frequency sub-bands. Thus, the number of zero coefficients in the shaft will be greater, object of the concept of the zero tree, which is to bring together the more coefficients of a single tree. Allowing a best encoding the significance map. We observe so that the best PSNR and image quality is for the decomposition level that $\mathrm{J}=3$.

\section{Influence of the choice of Quantization algorithm:-}

The following results shows a comparison between the two best algorithms (EZW and SPIHT) used in part of quantization applied for Toulouse satellite image for different compression ratio (see table 4 and figure 9).

Table 4:Comparison between EZW and SPIHT algorithms for different compression ratio

\begin{tabular}{lcrrrrrr}
\hline RC & RC 0\% & RC 10\% & RC 20\% & RC 30\% & RC 40\% & RC 50\% & RC 70\% \\
\hline EZW & 46,7 & 44,2 & 41,7 & 41,2 & 39,1 & 38,07 & 37,05 \\
SPIHT & 46,7 & 46,1 & 43,2 & 42,1 & 40,3 & 40,2 & 39,2 \\
\hline
\end{tabular}

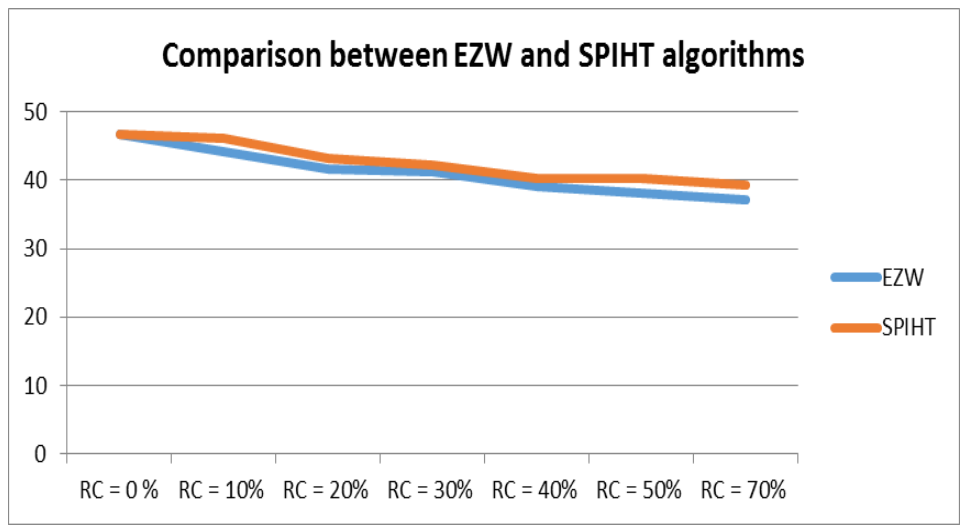

Fig 9: Comparison between EZW and SPIHT algorithms for different compression ratio in term of PSNR values 
This results shows that SPIHT algorithm performs better than EZW, this is justified by the SPIHT mechanism that average two trees Zerotree and better exploits the intra dependent-band by successive cutting the tree Zerotree.

\section{Comparison between DWT and DCT algorithm:-}

The following results shows a comparison between the DCT and DWT algorithm using the type of dB8 and Sym10 wavelet for different test images including the time execution (see table 5 and figure 10).

Table 5:.Comparison between DCT and DWT algorithms for different test images in term of PSNR values.

\begin{tabular}{|c|c|c|c|c|c|c|c|c|}
\hline Image & \multicolumn{2}{|c|}{$256^{*} 256$ gis } & \multicolumn{2}{|c|}{$1480 \div 858246$ its color } & \multicolumn{2}{|c|}{$1772^{2} 177224 \mathrm{bits}$ color } & \multicolumn{2}{|c|}{$4000 * 4000246$ its color } \\
\hline DCT & $0.32 \mathrm{~s}$ & 36,23 & $1.20 \mathrm{~s}$ & 33.42 & $1.90 \mathrm{~s}$ & 34.12 & $10.47 \mathrm{~s}$ & 34.74 \\
\hline DWT DB8 & $0,45 s$ & 28,53 & $1,41 \mathrm{~s}$ & 32.36 & $2.12 \mathrm{~s}$ & 39.62 & 10.065 & 43.57 \\
\hline DWT Sym 10 & $0.61 \mathrm{~s}$ & 28,93 & $1.64 \mathrm{~s}$ & 33.23 & $2.30 \mathrm{~s}$ & 40.98 & $11.20 \mathrm{~s}$ & 46.36 \\
\hline
\end{tabular}

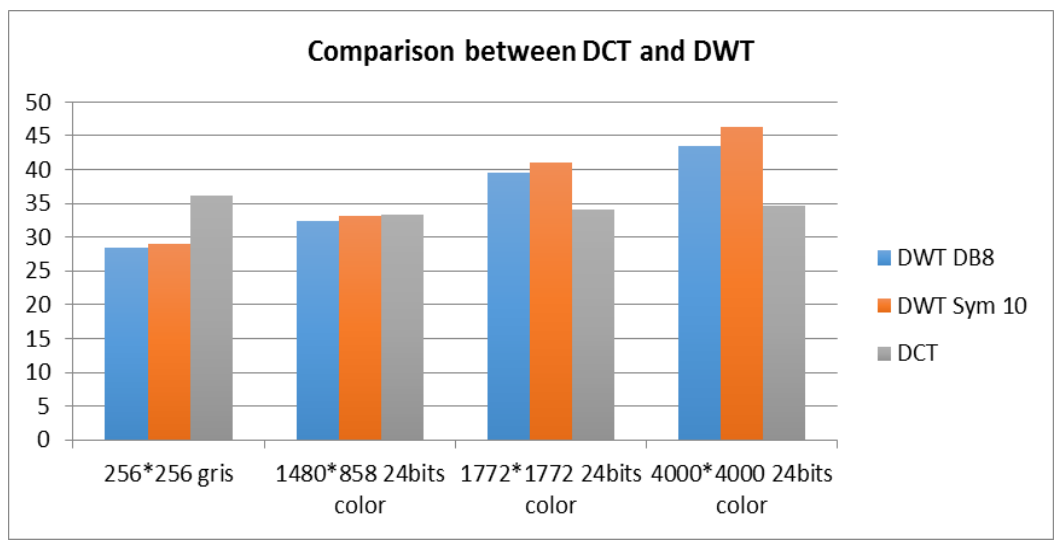

Fig 10: Comparison between DCT and DWT algorithms for different test images in term of PSNR values.

The comparison between the DCT and DWT method gives rise to a best actual record of the DCT algorithm on the smaller sizes of images with relatively medium compression rate, as to the compression process DWT proves its efficacy as images of large sizes, or high compression ratios, this is explained by the fact that the DCT undertakes the action of transformation on small block, and the picture is little better the performance, as to DWT transforms the image as a whole, it is clear thatthe processing of small images, does not lead to a better result than the DCT, as for large images as is the case of satellite images that characterized by high resolution, cutting the image into small block with the DCT, neglects the correlation infrared band, and therefore a lower compression ratio, in addition, if the user pushes the compression further, quality the image will last, from which the effect of image blocks which appears on the restored image. The following figure show the comparison between the DCT and DWT (Sym10 type of wavelet) according to the different values of compression ratio for Toulouse satellite image.

\section{Conclusion :-}

Through this work, we tried to test the parameters of the satellite image compression process, while trying to understand the impact of choosing each of these parameters on the final result of compression, appreciated to time factors, quality ( PSNR ), compression rate. It is more than obvious that each parameter to a present impact on rendering compression processing, starting with the choice of the type of wavelet for the case of DWT, or the finding was presented a few wavelets better results than others, then the level of decomposition, it determining that the level of the three is ideal to the choice of decomposition, also for the quantification method after comparison of the EZW and SPIHT two methods, choice is more oriented toward the SPIHT as seems to make better quality has averaged respect to the compression ratio.

Finally, simulations and results obtained allowed us to draw the most favorable parameters for a better quality factor/compression. One last comparison was made between DCT vs DWT to distinguish the performance of each method of transformation, and of course the DWT gives better results on high resolution images with a compression ratio pushed with maintaining a quality of acceptable image. 


\section{References:-}

1. Ken Cabeen and Peter gent, «Image compression and the DCT»,2003.

2. B. Penna, T. Tillo, E. Magli and G. Olmo, «Transform Coding Techniques for Lossy Hyperspectral Data Compression», IEEE Transactions on Geoscience and Remote Sensing, Vol. 45, 2007.

3. J. M. Shapiro, "Embedded Image Coding Using Zerotrees of Wavelet Coefficients», IEEE Transactions on Signal Processing, Vol. 41, 1993.

4. A. Bilgin, G. Zweig and M. V. Marcellin, «Three-Dimensional Image Compression with Integer Wavelet Transform», Applied Optics, Vol. 39, 2000.

5. A. Said, W.A. Pearlman, «A new fast and efficient image codec based on set partitioning in hierarchical trees», IEEE Trans. on Circuits and Systems for Video Technology., 6 (1996), pp. 243-250

6. L. Sunghyun, S. Kwanghoon and L. Chulhee, «Compression for Hyperspectral Images Using Three Dimensional Wavelet Transform», IEEE 2001 International Geoscience and Remote Sensing Symposium, Vol. $1,2001$.

7. Michel Misiti, Yves Misiti, Georges Oppenheim \& Jean-Michel Poggi, «Wavelets and their Applications», ISTE Ltd, 2007.

8. https://cnes.fr/ for Satellite test images.

9. Vijayabhaskara, «Comparison of wavelet filters in image coding using hybrid compression technique», International conference on nano electronics and embedded system, Jan 2013.

10. Chao Bei, Yujia Zhai, Jing Wu, «Efficient Wavelet-Based Compression for Hyperspectral Images Usingrecompression Algorithm », Multimedia andSignal Processing (CMSP), International Conference, vol 1, May 2012.

11. A. Karami, M. Yazdi, and G. Mercier, «compression of Hyperspectral images using discrete wavelet transform and tucker decomposition», IEEE Journalearth observations and remote sensing, vol. 5, no. 2, April 2012.

12. E.Christophe, C. Mailhes, and Pierre Duhamel 2008, «Hyperspectral Image Compression: Adapting SPIHT and EZW to Anisotropic 3-D Wavelet Coding», IEEETransactions on Image Processing, vol. 17, no. 12. 